\title{
Structure and evolution of 3D Rossby Vortices
}

\author{
S. Richard ${ }^{1, a}$ and P. Barge ${ }^{1, b}$ \\ ${ }^{1}$ Aix Marseille Université, CNRS, LAM (Laboratoire d'Astrophysique de Marseille) UMR 7326, 13388, \\ Marseille, France
}

\begin{abstract}
Three dimensional compressible simulations of the Rossby Wave Instability are presented in a non-homentropic model of protoplanetary disk. The instability develops like in the two dimensional case, gradually coming to the formation of a single big vortex. This $3 \mathrm{D}$ vortex has a quasi-2D structure which looks like a vorticity column with only tiny vertical motions. The vortex survives hundred of rotations in a quasi-steady evolution and slowly migrates inward toward the star.
\end{abstract}

\section{Introduction}

In two dimensions RWI has been studied in details both analytically [4] and numerically [3]. A chain of $2 \mathrm{D}$ vortices forms from the trapping of Rossby waves inside their corotation region, then it merges in a single vortex that commonly survives a large number of rotation periods. However, the formation and evolution of 3D vortices is a more complex issue than in 2D and the vortex structure depends on the vertical entropy gradient. Recent works already addressed the problem: (i) the analytical approach of Lin [3] when the instability is in the linear regime, (ii) the numerical simulations of Meheut et al. [3] when the flow is assumed homentropic (i.e) when the Brunt-Väisälä frequency $(N)$ vanishes in the whole disk. In this paper, we use 3D numerical simulations to revisit the problem starting from less restrictive assumptions than Meheut et al. [3]: we assume the disk is non-homentropic and the flow is adiabatic. In our study, the disk is stably stratified since $\mathrm{N}^{2}$ is always positive.

\section{Numerical simulation}

Simulations are performed with a code which solves the fully compressible Euler's equations with a second order finite volume scheme. Cylindrical coordinates $(r, \theta, z)$ are used and the standard numerical resolution is $200 \times 300 \times 100$. The computational domain is a complete annulus around the star: $5<r<10,0<\theta<2 \pi$ and $0<z<1$ where $r$ and $z$ are in AU. Symetric boundary condition are imposed at $z=0$. The equations we solve read:

$$
\frac{\partial \rho}{\partial t}+\vec{\nabla} \cdot(\rho \vec{V})=0
$$

\footnotetext{
a e-mail: samuel.richard@oamp.fr

be-mail: pierre.barge@oamp.fr
}

This is an Open Access article distributed under the terms of the Creative Commons Attribution License 2.0, which permits unrestricted use, distribution, and reproduction in any medium, provided the original work is properly cited. 


$$
\begin{gathered}
\frac{\partial \rho \vec{V}}{\partial t}+\vec{\nabla} \cdot(\rho \vec{V} \vec{V})+\vec{\nabla} P=\rho \vec{\nabla} \phi \\
\frac{\partial \rho e}{\partial t}+\vec{\nabla} \cdot((\rho e+P) \vec{V})=\rho \vec{V} \cdot \vec{\nabla} \phi
\end{gathered}
$$

where $\rho$ is the density, $\vec{V}$ le velocity, $P$ the pressure, $e=P(\gamma-1) / \rho+\vec{V}^{2} / 2$ the total energy of the gas and $\phi$ the gravitational potential of the star. The gas is assumed to be perfect and adiabatic. At steady state the unpertubed flow results from simple equilibria in the vertical and the radial directions:

$$
\begin{gathered}
\rho_{*}(r, z)=\rho_{0}\left(r / r_{o}\right)^{-\alpha} \exp \left(\frac{G M \mu}{k T_{*}(r)}\left(\frac{1}{\sqrt{r^{2}+z^{2}}}-\frac{1}{r}\right)\right) \\
T_{*}(r)=T_{0}\left(r / r_{o}\right)^{-q} \quad P_{*}(r, z)=\frac{k}{\mu} \rho_{*}(r, z) T_{*}(r) \\
u_{*}=0 \quad v_{*}(r, z)=\sqrt{\frac{G M r}{\left(r^{2}+z^{2}\right)^{3 / 2}}+\frac{r}{\rho_{*}} \frac{\partial P_{*}}{\partial r}} \quad w_{*}=0
\end{gathered}
$$

In the vertical direction, density and pressure have a nearly gaussian profile. In the radial direction density and temperature can be chosen as power laws (in the present work we set up $\alpha=2.75$ and $q=0.5$ ). The scale height of the disk is $0.5 \mathrm{AU}$ at $r=7.5 \mathrm{AU}$. The azimuthal velocity results from the radial balance between gravity, centrifugal force and pressure gradient. Then, initial conditions are chosen so as to change this equilibrium and makes it unstable. A simple way to get appropriate conditions is to start from an annular overdensity with the following relations:

$$
\begin{array}{r}
\rho(r, z)=\rho_{*}(r, z)(1+f(r)) \quad f(r)=A \exp \left(-\left(\frac{r-r_{0}}{\sigma}\right)^{2}\right) \\
T(r)=T_{*}(r) \quad P(r, z)=P_{*}(r, z)(1+f(r)) \\
u=0 \quad v(r, z)=\sqrt{\frac{G M r}{\left(r^{2}+z^{2}\right)^{3 / 2}}+\frac{r}{\rho} \frac{\partial P}{\partial r}} \quad w=0
\end{array}
$$

In the radial direction the overdensity has a gaussian profile with amplitude $A=0.2$ and width $\sigma=0.3$.

\section{Structure of the vortex}

In 3D, the instability develops in a similar way than in 2D: the annular overdensity fragments into a chain of vortices that merge into bigger and bigger vortices. When the instability start growing the number of vortices can be large. Their number depends on the azimuthal mode number of the perturbation initially injected for triggering the instability. Anyway, after a few tens rotations around the star, only one vortex remains. This vortex is long lived and behaves as a quasi-steady pattern. The structure of this vortex is presented in figure 1. It looks like a vorticity column in the disk thickness. Density and pressure have a gaussian profile in the radial and azimuthal directions (as in 2D), but also decays with height to respect the vertical balance between gravity and pressure gradient. In the meridional plane, the radial and azimuthal velocities have similar behaviour than for a $2 \mathrm{D}$ vortex. The vertical component of the velocity originates in the entropy gradient in the $\mathrm{z}$ direction. In our model the flow is an adiabatic one, so entropy is constant along the streamlines. The vortex can be assumed a quasi-steady pattern in a frame of reference rotating with the vortex center. In this frame of reference streamlines can also be considered as fluid particle trajectories. It means that fluid particles always stay on isentropic surfaces. Near $\mathrm{z}=1$, the vortex is slightly perturbed by boundary effect. 

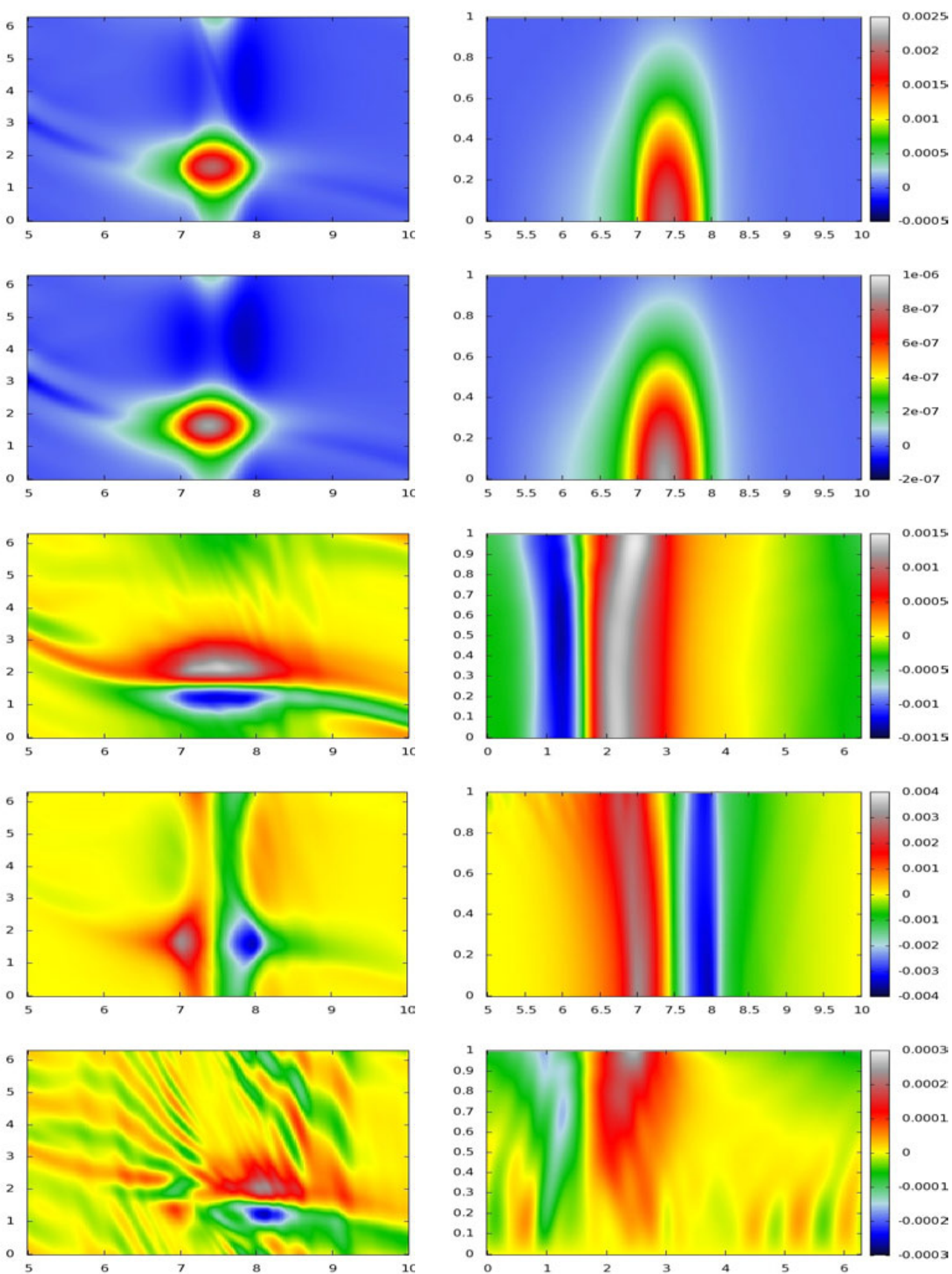

Figure 1. Three-dimensional structure of the vortex. From top to bottom the five rows represent: the density, the pressure, the radial velocity, the azimuthal velocity and the vertical velocity, respectively. The left column displays the values in the equatorial plane except for the vertical velocity which is displayed in the $(r-\theta)$ plane at $z=0.5$. The right column displays vertical profiles in the plane $(r-z ; \theta=2.67)$, except the third and fifth rows which display the radial and azimuthal velocities in the plane $(\theta-z ; r=7.5)$, respectively. 


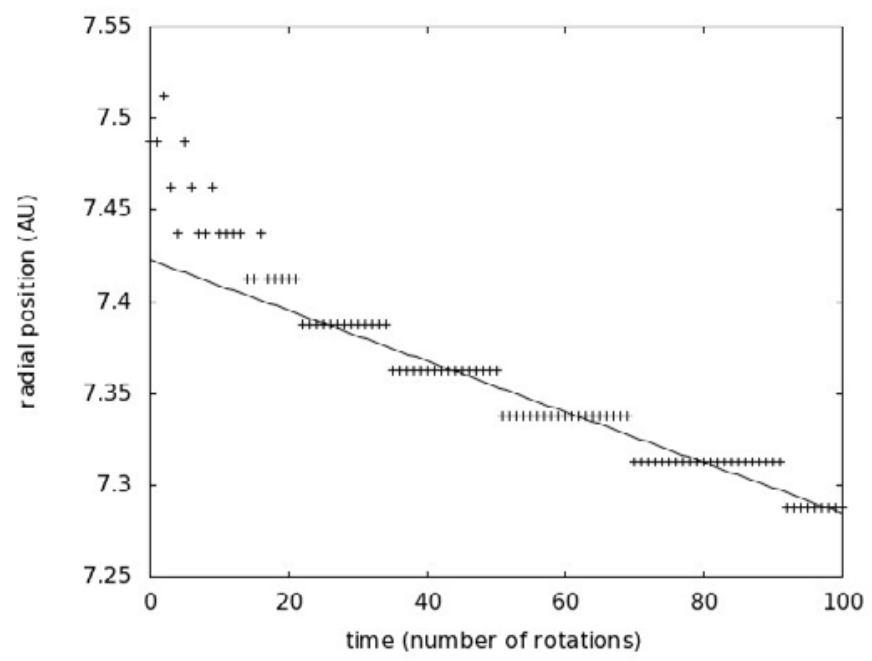

Figure 2. Radial position of the vortex as a function of time.

\section{Long term evolution}

In order to test the long term evolution of the isolated vortex formed at the end of the growth phase simulations were run starting from an approximate model of a Rossby vortex. This method avoid to simulate the quite long growth stage of the RWI and allows to significantly reduces the required CPU time. The 3D model we used is constructed from the 2D model developed by Surville \& Barge [3]. The obtained results are presented on Figure 2 which shows the radial position of the vortex as a function of time. We found that, after a short relaxation phase of a few rotations, the vortex remains unchanged and slowly migrates at a nearly constant rate equal to $6.810^{-2} \mathrm{AU} / 1000$ years or $0.126 \mathrm{AU} / 100$ rotations. The vortex can be considered as a quasi-steady structure since the ratio of the migration rate over the keplerian velocity also remains very small : $\dot{r}_{v} / v_{k}=5.510^{-4}$. These vortices survive for a very long time (hundreds of rotations) and seem to be unaffected by the elliptical instability (Lesur \& Papaloizou, ). This is likely due to the fact that the elliptical instability is most effective for small aspect ratios $(\chi<4)$ while our vortices have aspect ratios larger than 10. Simulations with a higher numerical resolution are also planned to test the impact of the smaller scales.

\section{References}

[1] Lesur G. \& Papaloizou J. C. B., A\&A 498, 1 (2009)

[2] Li H., Colgate S. A., Wendroff, B. B., \& Liska R., ApJ 551, 874 (2001)

[3] Lin M-K., ApJ 754, 21 (2012)

[4] Lovelace R. V. E., Li H., Colgate S. A. \& Nelson A. F., ApJ 513, 805 (1999)

[5] Meheut H., Casse F., Varniere, P. \& Tagger, M., A\&A 516, A31 (2010)

[6] Meheut H., Keppens R., Casse F. \& Benz W., A\&A 542, A9 (2012)

[7] Richard S. \& Barge P., work presented at the Ringberg Castle meeting - June 20-24, 2011

[8] Surville C. \& Barge P. arXiv:1201.3257v3 - French Congress of Mechanics 29th August 2011 